\title{
Architectural Planning Living with Robotics
}

\author{
Akiko Watanabe $^{1}$ and Yoshitaka Mikami ${ }^{2}$ \\ 1. Department of Architecture, School of Science and Technology for Future Life, Tokyo Denki University, Tokyo 120-8551, Japan \\ 2. Uchida Yoko Co., Ltd., Tokyo 104-8282, Japan
}

\begin{abstract}
This paper seeks to envision future architectural planning as it relates to living with robots and to clarify the research theme for it. Living with robots is no longer a fantasy seen just on TV or in movies. The concept of the intelligent space emerged from the field of robotics in the mid-1990s. The idea of the intelligent space is that the robot will support the architecture and users of the space by keeping in close contact with architectural space equipped with intelligent technologies. The benefit of the intelligent space is that the robot can be smaller and less intelligent because the robot will be assisted by advanced technologies embedded in the architectural space, such as sensors and actuators. Therefore, the robot can consequently assist user's activities with delicate care. This paper describes the vision and possibility for architectural planning as it relates to live with robots who can support the inhabitants' lives. This paper introduces the study of the relationship between humans and moving robot in architectural space, especially support region for humans by desktop mobile robot.
\end{abstract}

Key words: Architecture, robotics, planning.

\section{Introduction}

In recent years, machines and robots with certain types of intelligence and which intervene in environments inhabited by human beings have become more numerous, as they have the opportunities for themselves to assist human beings. However, there has not much research into the relationship between people in physical environments, i.e., architectural spaces, and artificial intelligent machines. In this paper, the authors examine the relationship between humans and robots which is expected to develop in architectural spaces in the future, and research is introduced in the area of robotic support of humans. The planning of architectural spaces is also discussed in which robots co-exist.

\section{Research Involving Robots and Architectural Spaces}

Research of robots has grown vigorously in the fields of intelligent information systems and robotics. Research and development of machines that control

Corresponding author: Akiko Watanabe, Ph.D., associate professor, research fields: architecture design and planning. E-mail: akiko826@cck.dendai.ac.jp. numerous sensors and actuators in the form of automatic machines has long been an active field, even if the machines were not called robots. In recent years, Prof. Kato et al. of Waseda University have combined computer technologies to create a robot called WABOT, which is capable of human-like behaviors. Following this development, Honda has created a machine capable of walking autonomously. The sight of machines walking has made the existence of intelligent machines more palpable.

Meanwhile, Nilsson [1] laid the foundations for research on robots which process sensor information and seek to understand the environment and converse with humans. This led to the development of wheeled intelligent robots used in many research institutions. Since then, research on computer vision that recognizes the environment and on intelligent functions that determines behaviors have grown.

In the field of architectural planning, Enta et al. [2] experimentally measured the distance needed to avoid robots when robots and people cross each other, and suggested that spaces in current home plans are too narrow.

As described above, researchers are creating 
situations in which artificially engineered objects such as robots communicate and live with people. As stated by Yamato [3], human beings living with robots are no longer a fantasy in the distant future.

Especially from the latter half of the 1990s, research on not just robots themselves but in connection with intelligent space in relation with robot, as the author wrote in design for the intelligent space has emerged [4].

\section{Architectural Space Living with Robotics}

The architectural space living with robotics seeks to create living spaces in which robots in intelligent architectural spaces exist and are connected to architectural spaces to provide better support for human beings and buildings (Fig. 1). In such living spaces, sensors are used to exchange information and connect humans and buildings, humans and robots, and robots and buildings to one another. In other words, an invisible virtual space on the information plane exists behind physical space, and in this virtual space, information is exchanged and linked between humans and buildings, humans and robots, and robots and buildings (Figs. 2 and 3).

Thinking about future architectural spaces in which robots co-exist with humans means that the themes dealt by architectural planning are inevitably changing.

\section{Support Region for Humans by Desktop Mobile Robot}

In recent years, the relationship between robots and humans has been researched gradually in architectural space. The "Basic Research on Support of Humans by Desktop Mobile Robots” project studied desktop mobile robots that carry out support services on desktops, such as approaching users when necessary during meetings and work tasks to notify them of information, and departing and standing by when they are not needed so as to avoid interfering with users. To study the region where users concretely felt they

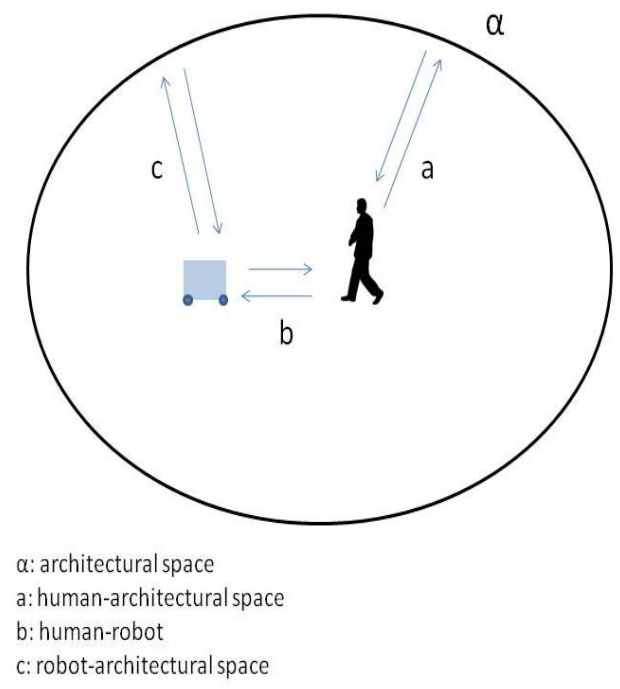

Fig. 1 Architectural space with robot 1.

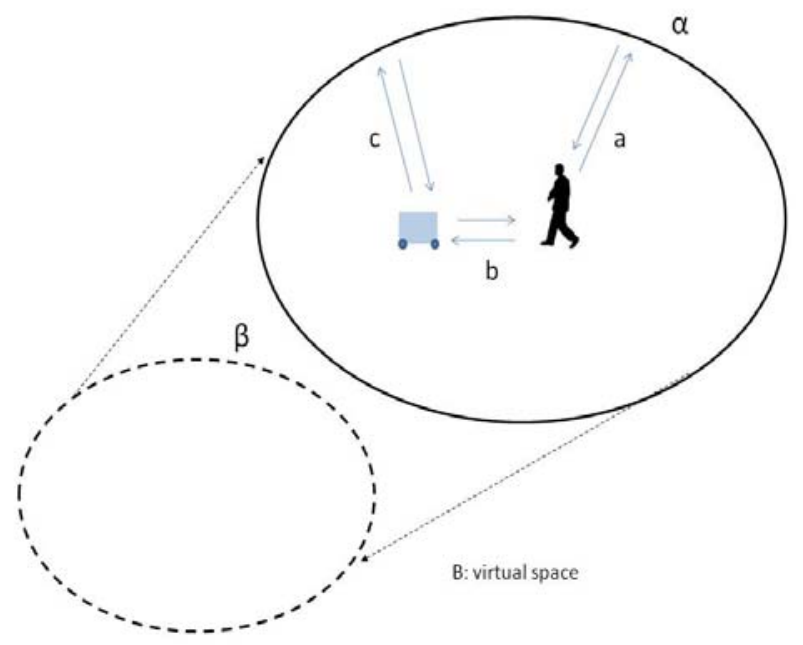

Fig. 2 Architectural space with robot 2.

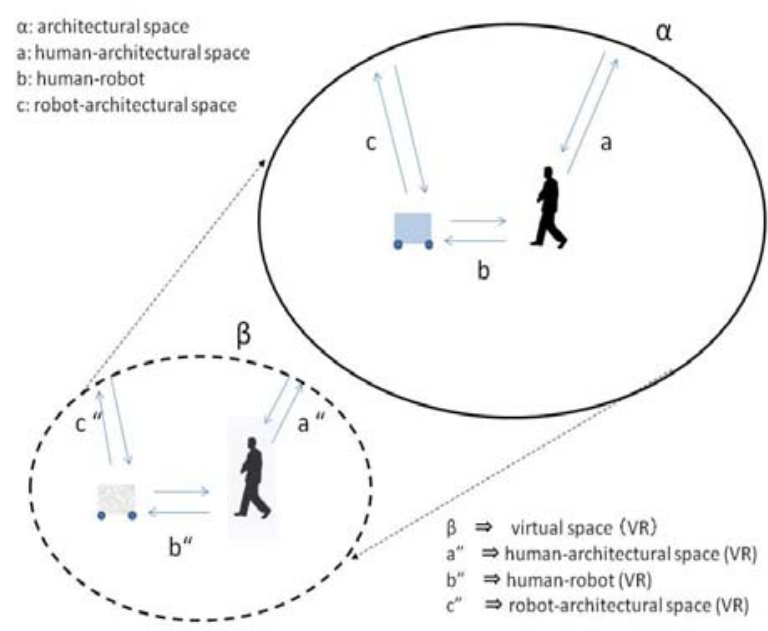

Fig. 3 Architectural space with robot 3. 
received support from desktop mobile robots, the authors defined the "proximity limit points" as the points where "if the robot approaches me beyond these points, I feel I could not receive support from it." The authors also defined the "support limit points" as the locations where "if the robot departs from me beyond these points, I feel I cannot receive support from it." The author defined the "support region" as the area between the "proximity limit points" and the "support limit points" where users felt they could concretely receive support from desktop mobile robots that seek to provide services. To determine the distances of the proximity limit points and the support limit points and the support region, the authors performed two experiments. Experiment 1 sought to determine the proximity limit points and Experiment 2 sought to determine the support limit points. To determine each of their distances, the authors established 15 types of conditions as shown in Table 1. Experiments 1 and 2 were both held on October 5 and 6 , 2006. The subjects were 33 university students ranging from age 18 to 26 (24 men, 9 women, average age 21.5). To get used to the robots and learn controls to a certain extent, the subjects practiced before the experiments and then moved to the experiments.

\subsection{Experimental Setup}

The experiments were carried out in a classroom on the campus of the university using the experimental setup shown in Fig. 4. Each part's details and configurations are shown in the figure. The subjects sit in a spot $300 \mathrm{~mm}$ from the lower left-hand corner of the desktop. From a reference point facing the subject, the robot approached the subject in Experiment 1 (1,500 $\mathrm{mm}$ at angles of 90 degrees, 67.5 degrees and 45 degrees, and 1,400 $\mathrm{mm}$ at angles of 22.5 degrees and 0 degree). In Experiment 2, the robot moved away from the subject from the reference point using the same distance and angles in Experiment 1. Fig. 5 shows a scene from the experiment. Now, the reference point was located at $300 \mathrm{~mm}$ from the lower left-hand corner of the desktop and $200 \mathrm{~mm}$ from its front edge. This was to prevent the robot from falling over. Taking into account the efficiency of the experiment, the boundary of measurement by the experiments were set as only entry from the right half of the desktop from the subject's point of view.

\subsection{Specification of Robot}

The robot used in the experiments was an autonomous mobile robot with two independent drive wheels. Its external dimensions were $110 \mathrm{~mm}(\mathrm{~W}) \times$ $120 \mathrm{~mm}(\mathrm{D}) \times 100 \mathrm{~mm}(\mathrm{H})$. The role of the robot in the experiments was to approach subjects to notify them of the arrival of personal information such as E-mail messages (Fig. 6).

\subsection{Method of Evaluation}

In this research, the movement speeds of the robot are one criteria to analyze proximity limit points and limit points. So the movement speeds of the robot were determined by preliminary experiments. Evaluations were carried out for low speed $(100 \mathrm{~mm} / \mathrm{s})$,

Table 1 Angle and speed.

\begin{tabular}{llllll}
\hline Speed & \multicolumn{5}{c}{ Angle } \\
\hline \multirow{2}{*}{$100 \mathrm{~mm} / \mathrm{s}$} & 90 & 67.5 & 45 & 22.5 & 0 degree \\
& degrees & degrees & degrees & degrees & \\
$250 \mathrm{~mm} / \mathrm{s}$ & 90 & 67.5 & 45 & 22.5 & 0 degree \\
& degrees & degrees & degrees & degrees & \\
$400 \mathrm{~mm} / \mathrm{s}$ & 90 & 67.5 & 45 & 22.5 & 0 degree \\
& degrees & degrees & degrees & degrees & \\
\hline
\end{tabular}

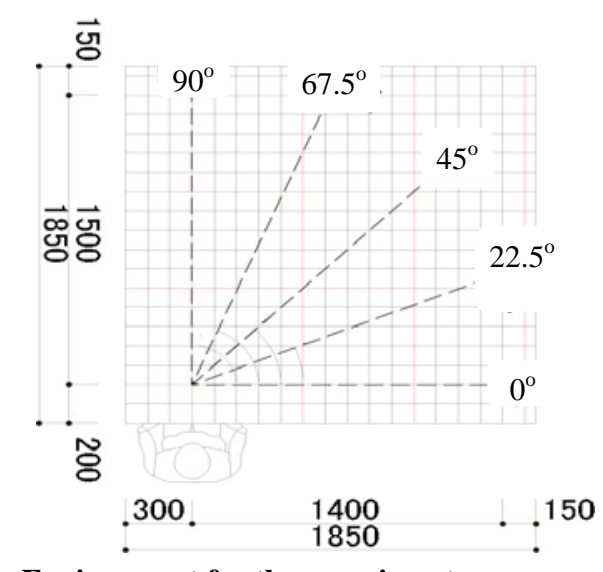

Fig. 4 Environment for the experiment. 


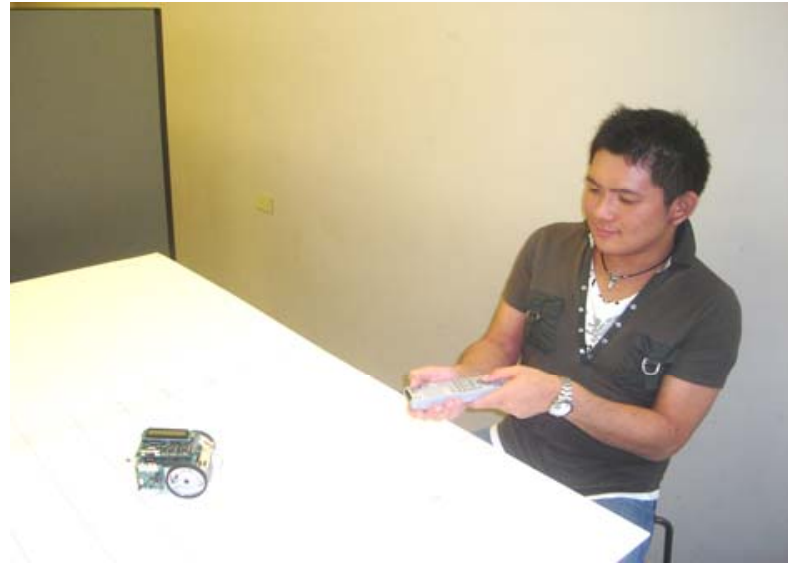

Fig. 5 Scenery of the experiment.

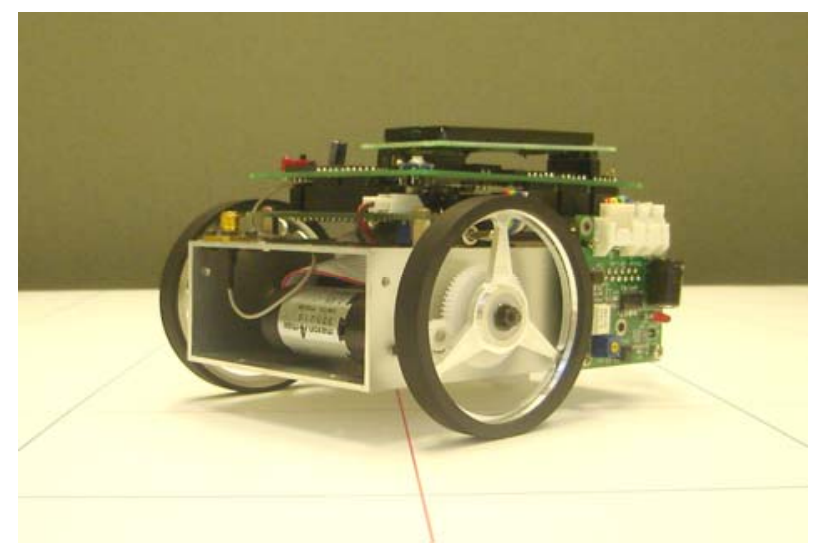

Fig. 6 Robot for the experiment.

medium speed (250 mm/s) and high speed $(400 \mathrm{~mm} / \mathrm{s})$. For Experiment 1, the subjects used a wireless remote to control switch to stop the robot at points where they felt that if the robot approached beyond those points, they could not receive support (the proximity limit points). For Experiment 2, the subjects also used the same method to stop the robot at points where they felt that if the robot moved away beyond those points, they could not receive support (the support limit points). After the robot was stopped by the subject, the distance from the reference point to the stopping point was measured. Now, the authors gave the following instructions to each of the subject concerning the experiment: "The role of the robot is to approach a person to notify him or her of the arrival of personal information, such as E-mail messages. Please do not consider the boundaries of the desktop. Do not change the direction of your body, or avoid the robot. Direct your line of sight on the robot. Do not evaluate the robot's state or the presentation of information."

\section{Results}

Data from each subject obtained from Experiment 1 were superimposed and shown in Fig. 7, along with the $95 \%$ confidence intervals and average values. For the gradation, the darker its shade was, the more the data obtained from the experiment was superimposed. This means an increase in the number of subjects who felt that they could not receive support from the robot when it approached beyond certain points.

From the average of the 95\% confidence intervals, the following distances were acceptable boundaries if they were not crossed: 90 degrees: $29.1 \mathrm{~cm}, 67.5$ degrees: $31.5 \mathrm{~cm}, 45$ degrees: $34.5 \mathrm{~cm}, 22.5$ degrees: $37.2 \mathrm{~cm}, 0$ degree: $42.3 \mathrm{~cm}$.

The authors were thus able to determine the proximity limit points (or the points beyond which if the robot moved closer to the subjects, the subjects felt they could not receive support) for the robot, whose goal was to approach users to provide support. Next, the data of Experiment 2 obtained from each subject were superimposed and shown in Fig. 8, along with the 95\% confidence intervals and average values. For the gradation, the darker its shade, the more the data obtained from the subjects was superimposed. This means an increase in the number of subjects who felt that they could not receive support from the robot when it moved away beyond certain points.

From the average of the 95\% confidence intervals, the following distances were acceptable boundaries if they were not exceeded: 90 degrees: $64.6 \mathrm{~cm}, 67.5$ degrees: $67.7 \mathrm{~cm}, 45$ degrees: $70.2 \mathrm{~cm}, 22.5$ degrees: 74.0, 0 degree: $78.5 \mathrm{~cm}$.

The authors were thus able to determine the support limit points (or the points beyond which if the robot moved away from the subjects, the subjects felt they could not receive support) for the robot. Also, by studying the mutual interactions between the direction of movement and speed, the authors found that when 


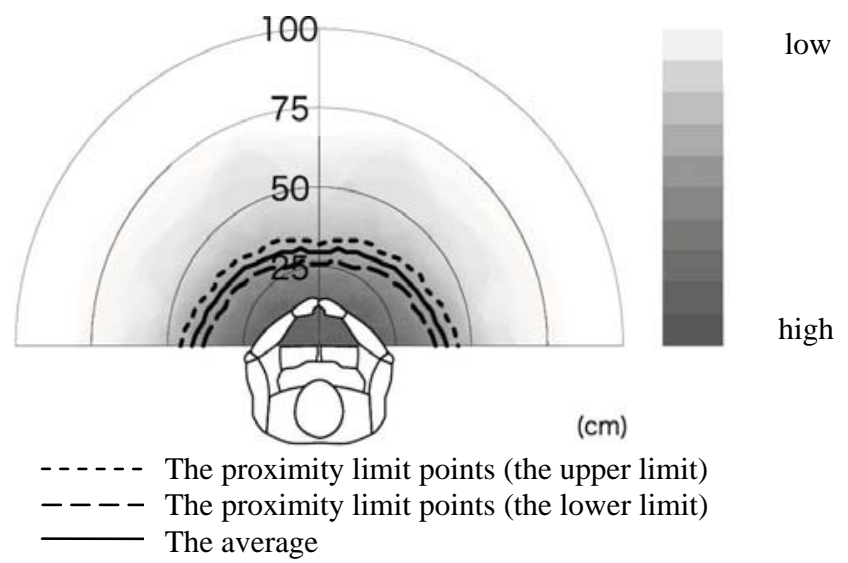

Fig. 7 The gradation from Experiment 1.

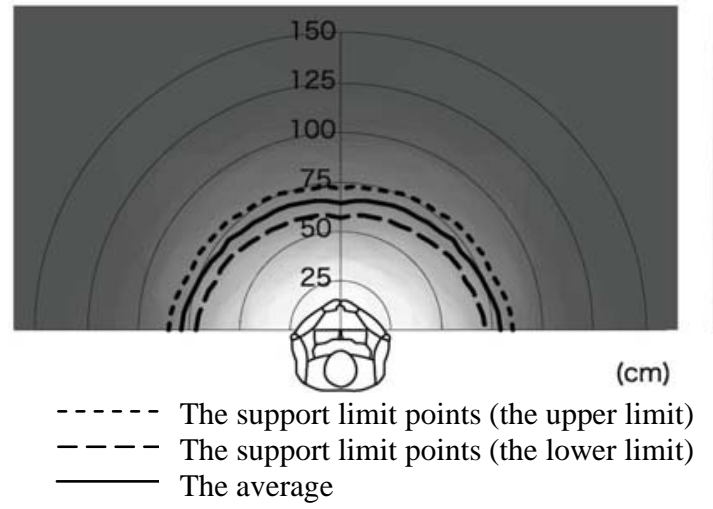

low

high

Fig. 8 The gradation from Experiment 2.

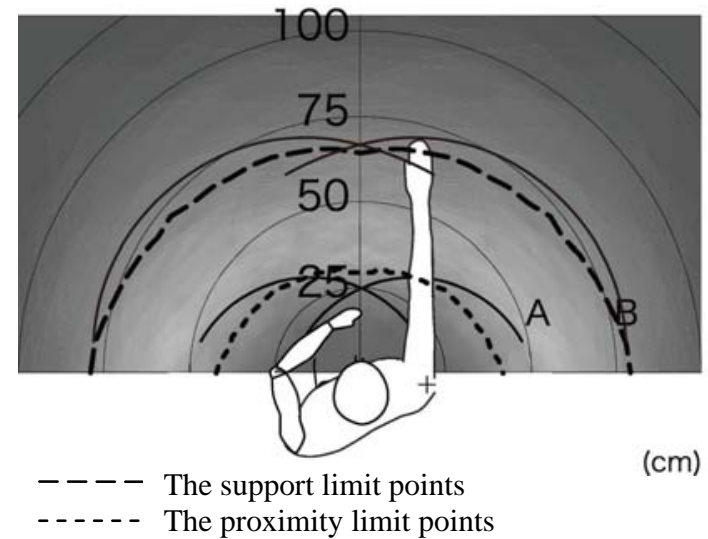

low

high

Fig. 9 The support from the robot: A. an arc traced by the user moving his or her forearms to the horizontal direction with the elbows as the fulcrums; B. an arc traced by the user placing his or her upper body on the desktop and moving his or her arms to a horizontal direction.

the robot approached the subjects, the degree of the movement had a greater effect than the speed of the movement. However, when it came to moving away from the subjects, speed had a greater effect. The faster the speed was, the greater the acceptable distance was, and the slower the speed was, the shorter the acceptable distance was.

The gradation showing the result of Experiment 1 and the average of its 95\% confidence intervals and the gradation of the results of Experiment 2 and the 
average of its $95 \%$ confidence intervals, together with the range of possible movement by a person seated were superimposed. The darker the shade of the gradation was, the more the data obtained from the experiment was superimposed. This means an increase in the number of subjects who felt that they could not receive support by the robot (Fig. 9).

When a subject felt that he or she could not receive support from the robot if it approached beyond a certain point, the boundary is similar to an arc traced out by the forearms moving to a horizontal position with the elbows as the fulcrums. This suggests a deep relationship between the length of the workspace and the distance between a person and the robot. When a subject felt that he or she could not receive support from the robot if it moved away beyond a certain point, the boundary was similar to the trajectory traced out by the user's upper body lying down and the arms moving to a horizontal position. This suggests that whether the hand can physically reach something has a deep relationship with a bodily sense of distance.

\section{Conclusions and Future Topics}

This paper described the vision and possibility for architectural planning as it relates to living with robots that can support the inhabitants' lives.

In this research project, the authors clarified the support region between the proximity limit points, or the points beyond which if the robot (whose goal is to provide support by approaching the user and notifying him or her of the arrival of personal information, such as E-mail messages) approached the user, the user would feel that he or she cannot receive support, and the support limit points, or the points beyond which if the robot moved away from the user, the user would not feel that he or she can not receive support.

The authors found that the distance for which the subjects sensed that they could not receive support as the robot approached them was affected by the direction of movement more than the speed of the movement. The region traced out was a horizontal oval.

This paper also found that the distance for which it was sensed that support could not be received as the robot moved away from the user was affected by both the speed of the movement and the direction of movement. If the robot's speed was faster, the user accepted a farther distance, and if the robot's speed was slower, the acceptable distance became shorter. The region traced out was also a horizontal oval.

The points beyond which subjects felt that they could not receive support as the robot approached them resembled an arc traced by the user moving his or her forearms to the horizontal direction with the elbows as the fulcrums. The points beyond which the subjects felt that they could not receive support as the robot moved away from the user resembled an arc traced by the user placing his or her upper body on the desktop and moving his or her arms to a horizontal direction.

In the future, information technologies and robotic technologies are expected to be incorporated inside architecture spaces and intelligentize the space. By connecting the intelligent space with mobile robots inside the space, better support services can be provided for the architectural space and for users of the space.

In this paper, the authors introduced research that concretely clarified the region in which mobile robots exist.

As shown in Fig. 9, the relation between human's movements can approach users and provide support services inside architectural space, especially on a desktop.

From the authors' study, the desktop mobile robot's support region has a close relationship with a person's the support region boundary of action. It led to the hypothesis that there is a close relationship between a bodily sense of distance and the workspace as a physical length of distance and whether the hand can physically reach something. 
In the future, the authors plan to carry out experiments including specific experiments on: (1) the relationship between humans in architectural spaces and the anthropometric dimensions of a mobile robot; (2) the usability of robots in architectural spaces; (3) robots in architectural spaces that provide services that include visual and other senses, to advance research on planning new architectural spaces in which humans and robots co-exist.

\section{References}

[1] N.J. Nilsson, Shakey the Robot, Techinical Note No. 323, SRI International, Menlo Park, CA, Apr. 1984.

[2] A. Enta, H. Watanabe, T. Sano, K. Hayashida, A study of avoidable distance against robot, Journal of Architecture and Planning 601 (2006) 81-85.

[3] N. Yamato, Roboto to Kurasu [Living with Robots], Soft Bank Book, Japan, 2006.

[4] H. Hashimoto, A. Watanabe, Design for the Intelligent Space, NTT Press, Japan, 2004. 